\title{
Cooperative Ant Colony Optimization in Traffic Route Calculations
}

\author{
Rutger Claes and Tom Holvoet
}

\begin{abstract}
Ant Colony Optimization (ACO) algorithms tend to be isolated processes. When applying ACO principles to traffic route calculations, ants exploring the traffic network on behalf of a vehicle typically only perceive and apply pheromones related to that vehicle. Between ants exploring on behalf of different vehicles little cooperation exists. While such cooperation could improve the performance of the ACO algorithm, it is difficult to achieve because ants working on behalf of different vehicles are solving different problems. This paper presents and evaluates a method of cooperation between ants finding routes on behalf of different vehicles by sharing more general knowledge through pheromones. A simulation of the proposed approach is used to evaluate the cooperative ACO algorithm and to compare it with an uncooperative version based on the quality of the calculated routes and the number of iterations needed to find good results. The evaluation indicates that the quality of the solution does not improve and that the speedup is insignificant when using the collaborative variant.
\end{abstract}

\section{Introduction}

Research on future Intelligent Traffic Systems (ITS's) assumes that a distributed ICT infrastructure embedded in the traffic network will be available [7]. Through this infrastructure, decentralized coordination of traffic can be made possible. By anticipating future traffic, situations leading to congestion can be avoided. Finding routes based on the forecast information is a challenge because the forecast information is often distributed across the traffic network.

Ant Colony Optimization (ACO) [5] is an optimization technique suitable for constructing solutions in problem domains with graph-like - possibly distributed - structures. In ACO algorithms, virtual ants construct solutions by exploring the graph representing the problem domain. As the ants roam the graph, they can sense and deposit pheromones allowing the ants to guide the probabilistic behavior of other ants exploring that same graph. Thus, knowledge of previous found solutions is embedded into the graph. Other ants then use this knowledge to guide them. This indirect means of communication and coordina-

\footnotetext{
Rutger Claes

Department of Computer Science, University of Leuven, Belgium, e-mail: rutger.claes@cs.kuleuven.be

Tom Holvoet

Department of Computer Science, University of Leuven, Belgium, e-mail: tom.holvoet@cs.kuleuven.be
} 
tion is called stigmergy. The use of stigmergy means ACO algorithms do not assume all information is in one central location, but rather assumes that it is distributed over a graph.

Because of these characteristics, ACO is suitable for solving the vehicle route calculation problem. Ants explore the traffic graph on behalf of the vehicle and construct routes to its destination. As the ants explore the graph, they aggregate information on the path they follow. This information, distance travelled and time travelled, can be used by the vehicle to choose one route.

Intelligent Traffic Systems (ITS) are capable of generating link travel time predictions, estimates of how long it will take a vehicle to traverse a link in the traffic network at a future time $[2,9,6]$. Most of the techniques used to generate these predictions are based on observations made by road side sensors. Scalable, robust ITS systems able to generate such link travel time predictions will probably be decentralized, embedding the link travel time predictions in the traffic network. ACO based algorithms are good candidates for making routing decisions based on these dynamic and distributed link travel time predictions. Earlier work [3] describes and evaluates such an ACO inspired algorithm. The algorithm is shown to outperform an $A^{*}$ algorithm operating on static network information by incorporating the link travel time predictions in the route calculation process.

Traffic routing is a composite problem: every vehicle in the system represents a different routing problem. Ants exploring the environment look for solutions to their problem, interpret pheromones describing previously found solutions to that problem and deposit pheromones describing how well their solution solves the problem. There is no cooperation between ants working on different problems, that is, there is no cooperation between ants finding routes to different locations.

Pheromones deposited by ants contain information on how well a route solves a certain problem. Because of the specificity of this information, ants trying to solve other problems cannot interpret this information. For ants to cooperate while finding routes to different locations, the information they exchange has to be more generic. The algorithm described in this paper establishes a common ground for ants representing different vehicles to cooperate. In this cooperative ACO algorithm, the ants not only deposit highly specific pheromone information relating to their own problem, they also deposit more general information about the routes they discover to aid other ants, possibly working on behalf of other vehicles. This general information is sufficiently generic to be of use in other route calculation problems.

The common ground for cooperation used in this approach is the concept of a region. Ants in search of routes will be interested in routes leading to locations that are near their destination. To achieve this, locations are grouped based on the region they are in. Regions are collections of locations near each other, such as all possible locations within one city.

When ants deposit pheromones representing the quality of the route they have constructed, they also deposit pheromones describing the different regions this route passes through. An ant, having found a route that passes a certain region, deposits pheromones informing other ants that its route leads to this region. Other ants, looking for routes to destinations in that region, can make use of these pheromones. The information embedded in the pheromones will guide other ants, possibly working on behalf of other vehicles. By improving the average quality of solutions constructed by ants, the algorithm will require less iterations to find meaningful results.

Reducing the number of iterations will reduce the number of ants sent out to explore the environment thus reducing the communication required to compute a solution. However, maintaining the additional region-specific information in the environment is an overhead 
compared to the earlier algorithm described in [3]. The aim of this paper's experiments is therefor to evaluate the cooperative extension, and investigate whether the usefulness of the additional information is worth the cost of gathering and maintaining it.

\section{Related work}

Biologically inspired algorithms and virtual pheromones are often used in the traffic routing domain $[8,1]$. However, most approaches either focus on solving the routing problem for one individual vehicle or focus solely on the self-organization of traffic by using pheromones to stochastically guide vehicles. This paper presents an algorithm in which ants are used to search for routes and where the traffic system piggybacks on the behavior of these ants to self-organize route guidance based on the cooperative pheromones. This section describes a number of other approaches that have similar characteristics to the approach presented in this paper.

In their paper [1], Ando et al. describes how pheromone communication between vehicles can be used to guide traffic and avoid traffic congestion. In the work of Ando et al., vehicles deposit pheromones as they travel through the traffic network. Pheromone levels will increase as more vehicles pass an intersection or vehicles slow down near the intersection. The pheromones spread through the edges of the traffic network, forming pheromone hotspots. The pheromones thus dispersed in the traffic graph repel other vehicles. Through this mechanism, vehicles use pheromones solely to inform each other of congestion in an indirect manner. They do not use pheromones to guide their own route calculation algorithm, as the vehicles using the cooperative ACO approach described in this paper do.

The cooperative aspect of the algorithm described in this paper resembles the ACO inspired AntNet routing mechanism for communication networks described by Di Caro and Dorigo in their work [4]. AntNet builds and maintains routing tables in the nodes of a communication network. The forward moving ants used in the mechanism travel trough the communication network like regular communication packets. This allows them to measure transfer times on the links they traverse. Having reached their destination, the forward ants are replaced by backward moving ants. These backward moving ants backtrack the path and adjust the routing tables on all encountered nodes.

This mechanism is very similar to the cooperative pheromones described in this paper, the AntNet algorithm was successfully applied to routing in traffic networks by Tatomir et al. in [8]. In the routing mechanism developed by Tatomir et al. ants explore the traffic graph similarly to the AntNet exploration of the communications network. At regular times ants are send from one random location to another and while searching for a path through the traffic graph, they accumulate information about the route. After reaching their destination, the ants backtrack their route and update a virtual stochastic routing table. Vehicles wanting to use the information left by the ants, can use the probabilities stored at every intersection to choose between which route to follow. The approach is hierarchical and uses a concept similar to the regions described in this paper to structure the locations and thus generalize the information contained in the pheromones. 


\section{ACO for traffic route calculations}

A description of how Ant Colony Optimization can be successfully applied to route calculation is given in our previous paper [3]. This section briefly outlines the approach allowing the next section to describe the cooperative extension to the approach. In [3] details on how the ACO approach presented in this section differs from other ACO-inspired algorithms such as Ant Colony System [5] or why these modifications where deemed necessary are also provided.

First we explain the graph representation used in the remainder of this paper. The traffic network is represented as a unidirectional graph in which edges $(i, j)$ connect vertices $i$ and $j$. Edges represent roads, Vertices crossroads. Bidirectional roads are represented by two edges, $(i, j)$ and $(j, i)$. Roads are characterized by their length, $l_{i j}$, and maximum speed $v_{i j}$. The set of outgoing edges at vertex $i$ is denoted as $O(i)$.

The link travel time for edge $(i, j)$, i.e. the time a vehicle would need to traverse the road represented by the edge $(i, j)$ assuming the vehicle travels at the maximum speed $v_{i j}$, is written as $l t t_{i j}$. Because of fluctuations in traffic, vehicles will not travel at the maximum speed $v_{i j}$. Instead, a vehicle arriving at the start of the road represented by edge $(i, j)$ will experience an average speed $v_{i j}(t)$ that is a function of its arrival time $t$. The actual time dependent link travel time is denoted as $l t t_{i j}(t)$. In this paper we assume that all vehicles entering a road at time $t$ will have the same $l t t_{i j}(t)$.

The benefit of using an ACO inspired route calculation algorithm is that the route calculation process can easily make use of link travel time predictions. By using predicted values for $l t t_{i j}(t)$, the route calculation algorithm can produce faster routes. Such a prediction of $l t t_{i j}$ at a future time $t$ is written as $\overline{l t t_{i j}}(t)$. Means of generating link travel time predictions are described in $[2,9,6]$.

The approach described in [3] relies on ants that explore the network on behalf of a vehicle. While traversing the graph, the ants keep track of a virtual time $t_{v}$ representing the time it would take the vehicle to reach the ants current location.

The ant constructs paths starting at the vehicles current location, vertex $o$, by selecting an edge $(o, i)$ from the set of outgoing edges at $o, O(o)$. Next, the ant will request a link travel time prediction $\overline{l t t_{o i}}\left(t_{v}\right)$. The ant will traverse the edge $(o, i)$ and move to vertex $i$. Using this link travel time the ant updates its virtual time value at $i$ to $t_{v}+\overline{l t t_{o i}}\left(t_{v}\right)$. The ant will then choose a next edge from the set of outgoing edges $O(i)$ at $i$ and repeat the process until eventually it reaches its intended destination. At that point, the virtual time $t_{v}$ will be a time dependent prediction of the arrival time of the vehicle at its destination if it were to follow the same path as the ant. This algorithm is described below.

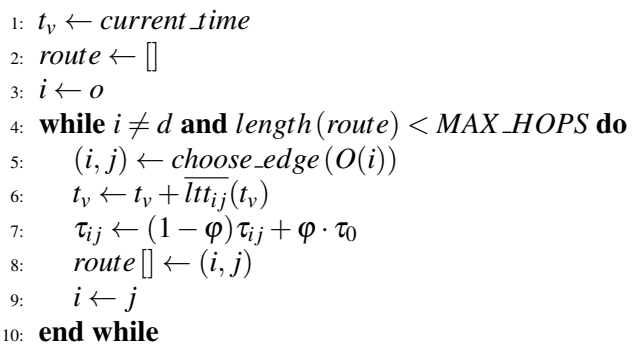

The edges $(i, j)$ in the graph have a pheromone level $\tau_{i j}$ associated with them. As an ant traverses an edge, it reduces the pheromone level on that path to discourage other ants to choose the same edge. The MAX_HOPS constant limits the ants lifetime. The 
choose_edge() function chooses the next edge using a stochastic method. The probability of an edge $(i, j)$ to be chosen by this method is given by:

$$
p_{i j}=\frac{(1-\gamma) \tau_{i j}^{\alpha} \cdot \gamma \eta_{i j}^{\beta}}{\sum_{(i, n) \in O}(1-\gamma) \tau_{i n}^{\alpha} \cdot \gamma \eta_{i n}^{\beta}} .
$$

Together, parameters $\alpha, \beta$ and $\gamma$ define a trade off between choosing the next edge based on pheromone levels $\tau$ or heuristic values $\eta$. The heuristic, $\eta_{i j}$ describing an edge $(i, j)$, is:

$$
\eta_{i j}=\frac{\|i d\|}{\|i j\|+\|j d\|}
$$

where $\|i, j\|$ is the euclidian distance between vertex $i$ and $j$.

If an ant manages to reach destination vertex $d$, it starts adjusting the pheromone levels of its constructed path while backtracking its previous movements. The backtracking is described as:

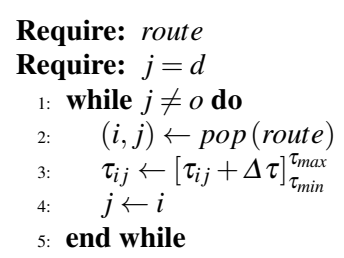

The $\left[\tau_{n l}+\Delta \tau\right]_{\tau_{\min }}^{\tau_{\max }}$ notation in the previous algorithm indicates that the result will be constrained by $\tau_{\min }$ and $\tau_{\max }$. The pheromone update, $\Delta \tau$, is given by: $\Delta \tau=\theta \frac{t t s_{\min }}{t t s_{\text {route }}}$, where $t t s_{\min }$ is the minimal travel time spent by a vehicle to get from origin $o$ to destination $d$ given by $\|o d\| / v_{\max }$ with $v_{\max }$ the highest allowed speed in the traffic network and where $t t_{\text {route }}$ is the travel time spent by a vehicle that would follow route, as constructed by the ant. $t t s_{\text {route }}$ is the difference between the current time and the virtual time $t_{v}$ of the vehicle when it arrives at $d$. The factor $\theta$ is a weighing factor.

The ACO inspired approach described in this section is an isolated, vehicle centric approach. Ants constructing a path on behalf of a vehicle $x$ only take into account pheromone values $\tau$ applied by other ants working on behalf of vehicle $x$. Vehicles with different origin and destination vertices have no use for each others pheromones, as these pheromones indicate the quality of an edge in function of their origin and destination vertices. The pheromones deposited by ants working on behalf of vehicle $x$, noted as $\tau(x)$, are therefor useless and probably even misleading for ants working on behalf of vehicle $y$. The algorithms from [3] presented in the previous section are therefore always scoped towards one single vehicle. Ants working on behalf of different vehicles never interact, not even through stigmergy. They each operate in a separate pheromone space.

\section{Cooperative ACO for traffic route calculations}

This section describes an extension on the isolated ACO algorithm described in the previous section. It allows vehicles' ants to partially use pheromones deposited by ants working on behalf of other vehicles. Taking this additional information into account should allow the cooperative ACO algorithm to find good solutions by sending out less ants as the ants, on 
average, find better routes. Considering the fact that, in a distributed system such as traffic, ants movements usually involve some communication, a reduction in ants needed to find a satisfying solution is significant.

The cooperative ACO approach described in this section employs a second kind of pheromones, cooperative pheromones. These cooperative pheromones $\sigma$ can be deposited and detected by ants working on behalf of different vehicles, thus allowing stigmergic collaboration between ants working for different vehicles.

Information conveyed by the "traditional" pheromones $\tau(x)$ of a vehicle $x$ is very specific and relates only to finding a route from that vehicles origin to that vehicles destination. In order for ants to interpret pheromones deposited by ants working on behalf of other vehicles, the information carried in the pheromones needs to be generalized. This generalization is achieved using regions. A region is a set of adjacent vertices in the graph.

Here we assume that not every vertex is part of a region and that regions do not overlap. Extending the region concept to include hierarchic regions or overlapping regions should be straightforward, however it falls outside the scope of this paper.

Ants constructing a path to a particular region will be guided by the pheromone levels $\tau$ as described in Section 3 and more specifically by Equation (1) as well as being guided by cooperative pheromones $\sigma$. These cooperative pheromones contain region specific information. Every vertex $i$ now holds two types of pheromones for every outgoing edge $(i, j)$ in $O(i)$. Vehicle specific pheromones $\tau_{i j}(x)$ for every vehicle $x$ that has passed and region specific pheromones $\sigma_{i j}(r)$ for every region $r$ reachable through that edge.

The cooperative pheromones are carried by backtracking ants. Ants passing a vertex $j$ in region $r$ while backtracking will pick up a pheromone payload much like bees pick up pollen while foraging. The pheromone payload is "sticky", it piggybacks along with the ant while it backtracks. Backtracking the edge $(i, j)$ after picking up the pheromone payload at $i$, some of the payload will be transferred on to the vertex $j$, where it will contribute to the $\sigma_{i j}(r)$ cooperative pheromone. As the ant travels further, the pheromone payload will diminish and the effect it has on local $\sigma(r)$ values will diminish. While backtracking, ants will carry pheromone payloads for all regions $r$ they have passed during the backtracking process. The $\sigma$ values themselves are also "sticky". Ants passing a vertex $i$ while backtracking will pickup $\sigma$-values for regions other than that of $i$.

Because of the cooperative pheromones and the pheromone payloads, ants will start building a gradient map for every region $r$ through which they travel. Future ants can make use of this gradient map and will reinforce it by carrying pheromone payloads as they backtrack. These gradient maps are vehicle agnostic, they do not describe the quality of a certain edge regarding the solution sought by one vehicle, instead they describe the quality of an edge regarding the region vehicles and thus ants are trying to reach. This additional information will help ants finding their target region faster, resulting in better routes constructed in less iterations.

While exploring the graph, ants use the cooperative pheromone levels to adjust the probability $p_{i j}$ of choosing an edge $(i, j)$ while at vertex $i$ :

$$
p_{i j}^{c}=(1-\lambda) p_{i j}+\lambda \sigma_{i j}(r) .
$$

In this equation, $r$ is the region the ant is trying to reach, $p_{i j}$ is the probability of choosing edge $(i, j)$ taken from (1) and $\lambda$ is a weighing factor between the vehicle specific and region specific information. For $\lambda=0$, region specific information is not taken into account and the ant will behave as described in Section 3 . 
The backtracking algorithm from Section 3 is modified to include the pheromone payloads:

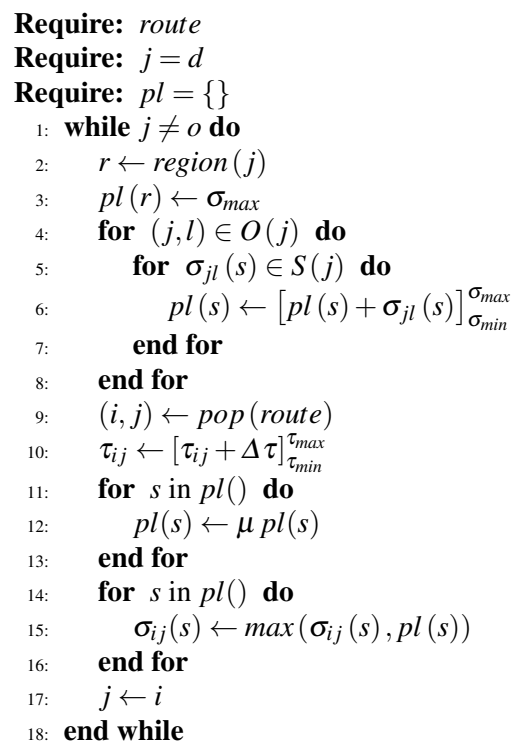

The variable $p l$ represents the pheromone payload carried along with the ant. Line 4 iterates over the outgoing edges of $j$ binding $l$ to the vertices reachable through these edges. The region () function will retrieve the region $j$ belongs to. $S(j)$ denotes the all $\sigma$ values present at $j$.

Lines 11 - 13 decrease the pheromone payload carried by the ant as it prepares to leave vertex $j$. Line 3 is the ant picking up the pheromone payload because of the region $r$ of the current vertex $j$. Lines $4-8$ is the ant picking up the pheromones in $S(j)$ and adding them to the pheromone payload. Lines $14-16$ is the ant depositing the pheromone payload and merging it into the $S(j)$ values at the destination vertex $i$.

\section{Evaluation}

This section evaluates the cooperative extensions described in Section 4 by comparing its performance with the original non-cooperating algorithm described in [3] and Section 3. The evaluation will focus on two aspects: (1) the quality of the final route found using the ACO algorithm and (2) the number of iterations needed to find high quality routes. The performance of both versions of the algorithm is evaluated using a simulation in a real-world traffic network. In this simulation a route is calculated for a number of vehicles based on link travel time predictions. The network layout is based on the OpenStreetMap data of Belgium. In this network, 21 regions are defined based on Belgian city regions. The network layout and regions are shown in Figure 1.

A set of 2048 origin destination combination was randomly generated so that every combination causes a trip between two different regions. The resulting origin destination matrix is used in all of the experiments. In every simulation these vehicles use either the cooperative or noncooperative ACO algorithm to find a route.

A Monte Carlo simulation was used to reestimate the parameters $\alpha, \beta, \gamma, \phi$ and $\theta$ described in [3]. A second Monte Carlo simulation was then used to estimate parameters $\mu$ 


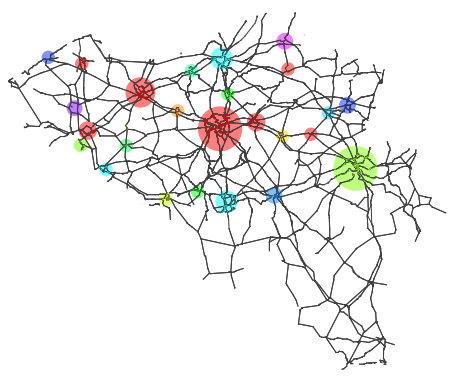

Fig. 1: The OpenStreetMap based network layout used to evaluate the performance of the non-cooperative and cooperative algorithm.

and $\lambda$. The resulting parameters are shown in Table 1 . The change in optimal parameters found by the Monte Carlo simulation in [3] and in this paper can be explained by the increase of the search space. Where the algorithms in [3] were evaluated based on citysized scenarios, the algorithms in this paper are evaluated on country sized scenarios.

\begin{tabular}{lr|c}
\hline \multicolumn{1}{c|}{ parameter } & value \\
\hline Pheromone power & $\alpha$ & 1.5 \\
Heuristic power & $\beta$ & 8 \\
Heuristic importance & $\gamma$ & 0.1 \\
Pheromone increase & $\theta$ & 8 \\
Pheromone decrease & $\varphi$ & 0.1 \\
\hline Cooperative importance & $\lambda$ & 0.1 \\
Pheromone range & $\mu$ & 0.8
\end{tabular}

Table 1: Parameter values used in the evaluation

Figures $2 \mathrm{a}-2 \mathrm{f}$ illustrate how the cooperative pheromones are spread through the environment. These figures show the distribution of $\sigma$ values on the edges surrounding a region. The figures show a distribution of $\sigma$ pheromones surrounding the cities, with strong intensities in the immediate surroundings of the city.

In order to evaluate the quality of a route $r_{o d}$ between an origin $o$ and a destination $d$ we compare the travel time on that route with the travel time on the optimal statically found route with the same origin and destination, $r_{o d}^{*}$. The optimal statically found route is the result of an $A^{*}$ search algorithm operating on the maximum speeds of the roads $v_{i j}$. When a vehicle traverses such a route, it will be influenced by traffic and will not reach $v_{i j}$. The route found by the $A^{*}$ algorithm is therefore no longer optimal. The quality $q(r)$ of a route $r_{o d}$ between $o$ and $d$ is given by: $q\left(r_{o d}\right)=\frac{\sum_{(i, j) \in r_{o d}} l t t_{i j}}{\sum_{(i, j) \in r_{o d}^{*}} l t t_{i j}}$.

The lower the $q(r)$ score of a route, the lower the duration needed for a vehicle to travel that route compared to the duration needed for the static route. Lower $q(r)$ scores are therefore better.

The graph in Figure 3a shows the evolution of the best found solution. This best found solution is defined as: $b f s(i)=\min _{j \in[0, i]}(q(r(j)))$, where $r(j)$ is the route found by the $j$-th ant. 


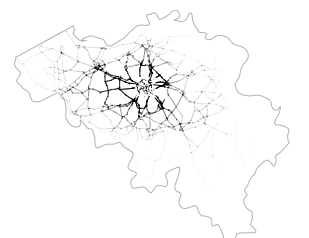

(a) Brussels

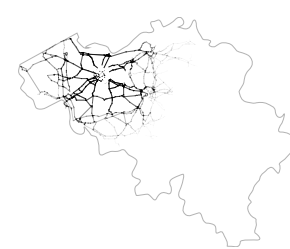

(d) Ghent

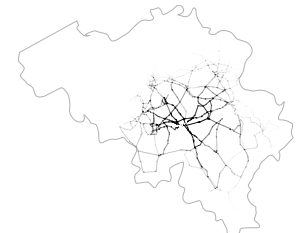

(b) Namur

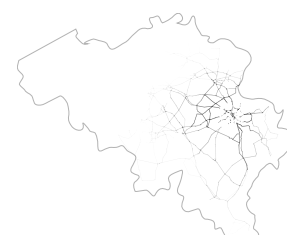

(e) Liège

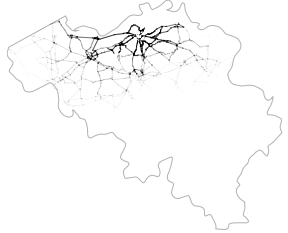

(c) Antwerp

Fig. 2: Spreading of the region specific pheromones for different regions.

Figure 3a shows that both the cooperative and non cooperative algorithms eventually find routes with similar qualities, but that the best cooperative algorithm finds better results faster. This observation is confirmed by Figure $3 \mathrm{~b}$ which compares, for every number of ants dispatched, the quality of the best found solution. Every dot in Figure $3 \mathrm{~b}$ represents a number of ants sent out by the vehicles. The values on the horizontal and vertical axes represent respectively the quality of the best known solution using the noncooperative and the quality of the best known solution using the cooperative algorithm. Most of the values fall below the diagonal, indicating that on average the quality of the cooperative solution is better.

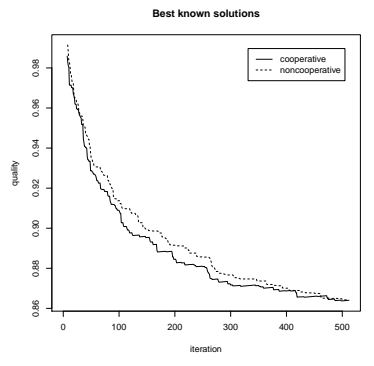

(a) Evolution of the quality of the best found solution

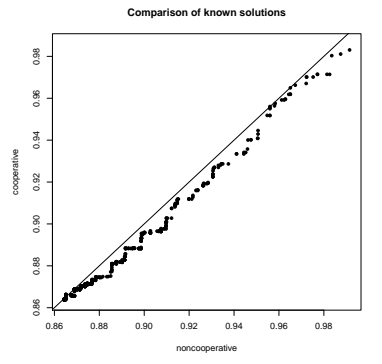

(b) Comparison of the quality of the best known solution

Fig. 3: Evolution and comparison of the best known solution in both approaches.

\section{Conclusion}

This paper presents a cooperative Ant Colony Optimization algorithm capable of simultaneously searching for solutions to individual vehicle problems using vehicle pheromones and cooperatively organizing route guidance through cooperative pheromones. The cooperative aspect of the approach results in less iterations needed to find qualitative results. 
Sending out less ants can have consequences on the communication requirements posed by distributed ACO based traffic routing mechanisms. However, maintaining the additional information needed for the region specific information poses an overhead compared to the original algorithm.

The benefit of using the additional information that is build up in the environment is limited to a reduction in iterations. The final solutions found by the cooperative and the original algorithms are comparable in quality. The reduction of iterations needed to arrive at these solutions is little, and for small networks will possibly be insignificant. However, considering that every iteration causes some inter-agent communication, the result is relevant.

The cooperative extension presented in this paper confirms the flexibility of ACO inspired algorithms and their applicability in traffic routing problems. By generalizing information, ants can share information they pick up while solving one routing problem and share it with all other ants working on other routing problems in the same distributed traffic graph.

Acknowledgements This research was funded by the IWT - SBO project 'MASE' (project no. 060823) and by the Interuniversity Attraction Poles Programme Belgian State, Belgian Science Policy, and by the Research Fund K.U.Leuven.

\section{References}

[1] Ando Y, Masutani O, Sasaki H, Iwasaki H, Fukazawa Y, Honiden S (2006) Pheromone model: Application to traffic congestion prediction. Engineering Self-Organising Systems pp 182-196

[2] Claes R, Holvoet T (2011) Ad hoc link traversal time predictions. In: Proceedings of the 14th International IEEE conference on Intelligent Transportation Systems, pp $1803-1808$

[3] Claes R, Holvoet T (2011) Ant colony optimization applied to route planning using link travel time predictions. In: 2011 IEEE International Symposium on Parallel Distributed Processing Workshops, pp 358-365

[4] Di Caro G, Dorigo M (1998) Antnet: Distributed stigmergetic control for communications networks. Journal of Artificial Intelligence Research 9(1):317-365

[5] Dorigo M, Gambardella L (2002) Ant colony system: A cooperative learning approach to the traveling salesman problem. Evolutionary Computation, IEEE Transactions on 1(1):53-66

[6] Fujimoto R, Hunter M, Sirichoke J, Palekar M, Kim H, Suh W (2007) Ad hoc distributed simulations. In: PADS '07: Proceedings of the 21st International Workshop on Principles of Advanced and Distributed Simulation, pp 15-24

[7] Maier M (1997) On architecting and intelligent transport systems. Aerospace and Electronic Systems, IEEE Transactions on 33(2):610 -625

[8] Tatomir B, Rothkrantz LJ, Suson AC (2009) Travel time prediction for dynamic routing using ant based control. In: Proceedings of the 2009 Winter Simulation Conference, pp 1069-1078

[9] Wunderlich K, Kaufman D, Smith R (2000) Link travel time prediction for decentralized route guidancearchitectures. IEEE Transactions on Intelligent Transportation Systems 1(1):4-14 